\title{
Memory for Angry Faces, Impulsivity, and Problematic Behavior in Adolescence
}

\author{
Mathieu d'Acremont • Martial Van der Linden
}

Published online: 23 January 2007

(C) Springer Science+Business Media, LLC 2007

\begin{abstract}
Research has shown that cognitive processes like the attribution of hostile intention or angry emotion to others contribute to the development and maintenance of conduct problems. However, the role of memory has been understudied in comparison with attribution biases. The aim of this study was thus to test if a memory bias for angry faces was related to conduct problems in youth. Adolescents from a junior secondary school were presented with angry and happy faces and were later asked to recognize the same faces with a neutral expression. They also completed an impulsivity questionnaire. A teacher assessed their behavior. The results showed that a better recognition of angry faces than happy faces predicted conduct problems and hyperactivity/inattention as reported by the teacher. The memory bias effect was more pronounced for impulsive adolescents. It is suggested that a memory bias for angry faces favors disruptive behavior but that a good ability to control impulses may moderate the negative impact of this bias.
\end{abstract}

Keywords Problematic behavior - Adolescence - Memory bias $\cdot$ Emotional faces $\cdot$ Impulsivity $\cdot$ Social information processing

Research in cognitive psychopathology has highlighted deficits and biases in relationship with the development and

\footnotetext{
M. d'Acremont ( $\square)$

Swiss Centre for Affective Sciences, University of Geneva,

Rue des Battoirs 7,

CH-1205 Geneva, Switzerland

e-mail: mathieu.dacremont@pse.unige.ch

M. Van der Linden

Cognitive Psychopathology and Neuropsychology Unit,

University of Geneva,

Switzerland
}

maintenance of conduct problems. More particularly, it has been found that aggressive youths recall fewer cues that are relevant to a social situation (Lochman \& Dodge, 1994), request less information before judging a social situation (Dodge \& Newman, 1981), and generate fewer solutions to interpersonal problems (Slaby \& Guerra, 1988). In addition to these cognitive deficits, numerous studies have reported cognitive biases in youths with conduct problems. For instance, aggressive children and adolescents are prone to attributing hostile intentions to peers whose behavior is ambiguous, and a meta-analysis suggests that this attribution bias is a robust finding (Orobio-de Castro, Veerman, Koops, Bosch, \& Monshouwer, 2002). More recent studies have shown that aggressive children also attribute angry emotions to others (Schultz, Izard, \& Bear, 2004). In addition to these attribution biases, attentional biases have also been associated with aggressive behavior. Among children, Gouze (1987) reported that aggressive boys paid more attention to aggressive than to cooperative interactions (however for conflicting results, see Schippell, Vasey, Cravens-Brown, $\&$ Bretveld, 2003).

Some of these empirical findings have been integrated by Crick and Dodge (1994) in an influential model of aggressive behavior. In this model, when an individual enters a social situation, information is processed in five steps before an action is taken: (1) encoding of cues, (2) interpretation of cues, (3) clarification of goals, (4) response access or construction, and (5) response decision. At each step, information may be encoded into, and retrieved from, one's cognitive database or memory storage. This database contains scripts, schemas, and social knowledge that guide the processing of information and increase its efficiency. However, knowledge stored in memory can also bias social information processing and result in social maladjustment. Indeed, it has been found that a negative conception of others or a poor understanding of 
emotion is related to hostile attribution bias and to aggressive behavior in children (e.g., Dodge, Laird, Lochman, Zeli, \& Conduct Problems Prevention Research Group, 2002). Because knowledge stored in memory is considered to guide the processing of social information in Crick and Dodge's (1994) model, one can make the hypothesis that the encoding of information in memory and its subsequent retrieval are also biased in aggressive children and adolescents, as are other social information processes such as the attribution of intention or the allocation of attention. For instance, a negative conception of the social world could favor the encoding of hostile information such as insults or anger expressed by others (step 1 of the model). Subsequently, a developing tendency to retrieve hostile information could favor a negative interpretation of new social situations (step 2), promote aggressive goals (step 3), favor the construction of aggressive responses (step 4), and increase the probability of an antisocial decision (step 5).

Three studies have assessed the recall of hostile information in aggressive children. Dodge and Newman (1981) asked boys to listen to a story about another boy who might have committed a hostile act and then to gather information that could help them to decide whether the boy actually committed the act. For example, one story concerned an incident in which "an eldery woman called a school to complain that on the previous day, a student had deliberately spilled her groceries and then had run away" (p. 376). After the participant heard the story, he was told that he could listen to audiotape testimonies that would help him to decide whether the suspect had committed the act. Testimonies consisted of sentences that either favored or rejected the possibility of a hostile act (supporting and nonsupporting statements). An example of a supporting statement for the previous story is "I saw him running in the direction of that woman's house yesterday after school" (p. 376). The results revealed no significant difference between the groups of aggressive and nonaggressive boys with regard to the proportion of supporting acts recalled. In a second study, aggressive and nonaggressive boys were shown the video of an interview. In this interview, an adult asked a boy to describe things that he did with kids in his classroom. The boy made hostile, neutral, and benevolent statements (Dodge \& Frame, 1982). An example of hostile statement is "This morning I kicked a boy named Joshua because he is ugly" (p. 625). Following the presentation of the interview, the participant was asked to recall as much as possible of what the boy in the video had said. Instances of correct recall of benevolent acts, neutral acts and hostile acts were counted. Nonsignificant differences were found between the groups in terms of the memorization of hostile information. In a third study, Milich and Dodge (1984) used both the testimony and interview tasks to compare groups of boys with and without aggressive behavior. Here again, non-significant differences were found between the groups in terms of the memorization of hostile information. Thus, none of these studies highlighted statistically significant results when aggressive and nonaggressive boys were compared. However methodological issues might explain this absence of significant differences. Firstly, the use of verbal statements is problematic for the study of conduct problems because these later are often associated with verbal difficulties (Teichner \& Golden, 2000). Secondly, it should be noted that in the testimony task, participants memorized statements supporting a hostile act, but the statement itself did not necessarily depict a hostile action (e.g., running in the direction of a woman's house is not a hostile action by itself). Thirdly, in the three studies, aggressive and control groups were formed by dichotomizing continuous variables (median split or extreme scores). This practice has various negative consequences, including the loss of information, power, and effect size (MacCallum, Zhang, Preacher, \& Rucker, 2002). As a consequence, it might be premature to conclude that conduct problems are not related to a memory bias for hostile information.

To overcome the limitations of previous studies, we used a memory task with emotional faces. Faces have the advantage of being visual stimuli, and performance at the task should depend less on verbal difficulties that are known to correlate with conduct problems. In addition, they are highly significant social stimuli. Indeed, of all the information available in a social situation, faces and emotional expressions are probably the most salient and important cues when one engages in social interaction (Bruce \& Young, 1986). During the encoding phase of the task, faces were presented with a happy or an angry expression. During the recognition phase, which took place five minutes later, the same faces were presented among new ones but all faces had a neutral expression. After seeing each face, the participant had to indicate whether it had been presented before. If the face was recognized, the participant had to remember whether its initial expression had been angry or happy. In our study, the recognition of faces' identity was separated from the recall of facial expressions. This procedure has several advantages. Firstly, current models of face processing generally assume that identity recognition and expression recognition involve independent processes. Secondly, it is probably a more ecological procedure because people generally express anger or happiness for only a short time (Bruce \& Young, 1986).

In order to assess adolescent behavior, a teacher completed the Strengths and Difficulties Questionnaire (SDQ, Goodman, 1997). This scale allows the evaluation of four types of problematic behavior: Conduct problems, Hyperactivity/Inattention, Emotional symptoms and Peer problems. The fifth dimension of the scale evaluates prosocial behavior. It should be noted that the Conduct problems subscale of the SDQ covers various types of antisocial behavior, including aggressive behavior, displays of anger, and 
oppositional behavior. The literature on social information processing has highlighted a hostile attribution bias in children with aggressive behavior, but also among children with oppositional defiant disorder (Webster-Stratton \& Lindsay, 1999). Because Conduct problems in the SDQ cover both aggressive behavior and oppositional behavior, we hypothesized that a higher score on this subscale would be related to a memory bias for angry faces.

Several years of research in the field of personality have highlighted impulsivity as an important predictor of conduct problems in adolescence (e.g., Luengo, Carrillo-de la-Pena, Otero, \& Romero, 1994). In addition, impulsivity seems to play an important role in the processing of social information. Dodge and Newman (1981) observed that aggressive boys responded faster and with less attention to available social cues than nonaggressive boys. Their results also showed that aggressive boys overattributed hostility to peers only when they responded quickly. Overall, these results suggest that the hostile attribution bias in aggressive children increases with their level of impulsivity. In adolescence, Colder and Stice (1998) have shown that trait anger is related to more delinquency and that this relationship is stronger for individuals with high impulsivity scores. These authors proposed that impulsive adolescents who frequently experience anger are more likely to act antisocially because they do not reflect on the consequences of their behavior. Thus, the effect of anger on social adjustment may be potentiated by impulsivity. Similarly, a memory bias for hostile information may have a greater impact on behavior among adolescents with a high level of impulsivity. With this in mind, the question arises whether impulsivity acts as a moderating variable on the hypothesized relationship between a memory bias for angry faces and conduct problems.

However, it should be noted that impulsivity is a heterogeneous concept (Gerbing, Ahadi, \& Patton, 1987). In a recent effort to clarify this concept, Whiteside and Lynam (2001) developed the UPPS Impulsive Behavior Scale, which has the advantage of being derived from previous impulsivity scales. The UPPS assesses four traits of impulsivity. The first trait, Urgency, "refers to the tendency to experience strong impulses, frequently under conditions of negative affect" (Whiteside \& Lynam, 2001: 685). The second trait, Premeditation, "refers to the tendency to think and reflect on the consequences of an act before engaging in that act" (p. 685). The third trait, Perseverance, "refers to an individual's ability to remain focused on a task that may be boring or difficult" (p. 685) Finally, Sensation seeking "incorporates two aspects: 1) a tendency to enjoy and pursue activities that are exciting and 2) an openness to trying new experiences that may or may not be dangerous" (p. 686). The different facets of impulsivity appear to be related to specific problems in adolescence. Lack of premeditation has been found to be the aspect of impulsivity that best predicts adolescent conduct problems reported by teachers with the SDQ (d'Acremont, 2005). Premeditation in the UPPS impulsive behavior scale seems to reflect an ability to take the consequences of an action into account (Zermatten, Van der Linden, d'Acremont, Jermann, \& Bechara, 2005) and it could incite adolescents to avoid antisocial actions that are followed sooner or later by negative consequences (e.g., legal sanctions). Lack of Perseverance has been found to be the aspect of impulsivity that best predicts Hyperactivity/Inattention (d'Acremont, 2005). This later result suggests that attentional processes are involved in the Perseverance facet of impulsivity. Further research is needed to identify the cognitive processes involved in the various facets of impulsivity. However, the ability to evaluate the consequences of an action (Premeditation) and the ability to control one's attention (Perseverance) may both help adolescents to reduce the negative impact of biased memories.

To sum up, one major contribution of previous research on conduct problems in youth has been to show how cognitive factors contribute to their etiology and maintenance. Encoding, attentional, and memory retrieval processes, however, have been understudied in comparison with attributional processes. A few studies have explored the memorization of hostile information but their results revealed no statistically significant bias in aggressive children. The absence of significant results may be explained by methodological limitations, mainly the use of verbal statements that are not clearly hostile and the dichotomization of continuous variables to form aggressive and control groups. To overcome these limitations, we used a memory task with angry and happy faces, which are highly relevant social stimuli. In addition, conduct problems was treated as a continuous variable to avoid the loss of information, power, and effect size. We also included an assessment of impulsivity because according to previous studies, social information processing bias seems to be more pronounced in impulsive children. Our main prediction was that in a community sample of adolescents, conduct problems would be related to a better memorization of angry faces. We also made the hypothesis that the memory bias effect would be moderated by impulsivity.

\section{Method \\ Participants}

The participants were students from a public junior secondary school located in Geneva, a city in the Frenchspeaking part of Switzerland. The school was selected by the Geneva administration that authorizes research projects in schools. To balance the sample, two classes were selected from the seventh grade, two from the eighth grade, and two from the ninth grade. There were 110 students in the six 
classes. Their parents were informed by mail about the study and their authorization was requested. The teenagers were free to choose whether to participate in the research and no compensation for participation was given. Ninetyfive students received authorization from their parents and chose to participate to the study. The students completed the face memory task collectively with their classes. Each student's teacher completed the SDQ for him or her.

Adolescents whom their teachers had known for less than 6 months were not included in the analysis. To reduce the risk of misunderstanding items of the UPPS Scale, nonnative speakers of French who had spoken the language for less than 5 years were not included. The validation studies of the French SDQ and UPPS indicated that the reliability of their subscales ranged from "acceptable" to "very good" (see below for the psychometric properties of the questionnaires). It was therefore reasonable to replace a missing value by the mean of the subscale that the particular missing value belonged to (Schafer \& Graham, 2002: 158). The final sample was made up of 86 students ( 36 girls and 50 boys) aged from 13 to 17 years old $(M=14.31, S D=0.99)$. Seventy-four students were citizens of European countries (including 43 Swiss citizens), 7 of African countries, 3 of Asian countries, 1 of Haiti, and 1 of Russia. Sixty-three were native French speakers, and the others had spoken French for a mean of 8.96 years $(S D=2.99)$.

UPPS impulsive behavior scale (UPPS, Whiteside \& Lynam, 2001)

The UPPS Impulsive Behavior Scale is an English questionnaire intended to assess four impulsivity traits: Urgency, lack of Premeditation, lack of Perseverance, and Sensation seeking. Answers are given on a Likert scale ranging from "Disagree strongly" (1) to "Agree strongly" (4). The scale was developed with American undergraduate students (Whiteside \& Lynam, 2001). It was adapted to French using a back-translation method and the four dimensions of impulsivity were confirmed in a sample of undergraduates (Van der Linden et al., 2006). It was then validated in a community sample of French-speaking adolescents that included the secondary school students in the present study (d'Acremont \& Van der Linden, 2005). In this latter study, the four-factor model of impulsivity was confirmed separately for boys $(n=314)$, girls $(n=314)$, and the whole sample by means of structural equation modeling. For the whole sample, the fit indices were SRMR $=0.068$ and RMSEA $=0.051$, which corresponded to an acceptable model (Schermelleh-Engel \& Moosbrugger, 2003). Internal reliability was .81 for Urgency (12 items), .82 for Premeditation (11 items), .83 for Perseverance (10 items), and .84 for Sensation seeking (12 items). Thus, the UPPS subscales appeared to have very good internal reliability $(>.80)$.
Strengths and difficulties questionnaire (SDQ, Goodman, 1997)

The SDQ is an English questionnaire intended to assess child and adolescent behavior (Goodman, 1997). The informantrated version of the questionnaire was used here. Answers are given on a 3-point Likert scale: "Not true" (0), "Somewhat true" (1), and "Certainly true" (2). The English scale has been validated among community samples of children and adolescents (Goodman, 2001) and makes a distinction between five types of behavior: (1) Conduct problems, with items assessing temper tantrums, disobedience, fighting or bullying, lying or cheating, and stealing; (2) Hyperactivity/Inattention, with items assessing restlessness, fidgeting or squirming, distraction or mind-wandering, absence of deliberation, and lack of perseverance; (3) Peer problems, with items assessing social isolation, lack of friendship, low popularity among peers, victimization, and relationships with adults rather than peers; (4) Emotional symptoms, with items assessing physical symptom complaints, worries, unhappiness, nervousness and fear; and (5) Prosocial behavior with items assessing consideration for others, sharing, helpful attitude, and kindness. The scale was translated into French using a backtranslation procedure (Fombonne et al., 2005). It was then validated in a community sample of Frenchspeaking adolescents that included the secondary school students of the present study (d'Acremont \& Van der Linden, 2006). The five-factor organization of the SDQ was confirmed separately for boys $(n=226)$, girls $(n=226)$, and the whole sample by means of structural equation modeling. For the whole sample, the fit indices were SRMR $=0.085$ and RMSEA $=0.058$, which corresponded to an acceptable model. Internal reliability was .75 for Conduct problems (5 items), .84 for Hyperactivity/Inattention (5 items), .62 for Peer problems ( 5 items), .76 for Emotional symptoms (5 items), and .89 for Prosocial behavior (5 items). Thus, the SDQ subscales appeared to have good to very good internal reliability $(>.70$ and $>.80)$, except for Peer problems which had an acceptable internal reliability (>.60).

\section{Emotional face memory task}

The procedure for the memory task was adapted from D'Argembeau, Van der Linden, Etienne, and Comblain (2003). Colored pictures of 24 faces, each displaying a neutral, a happy, and an angry expression, were used. These faces were selected from the Karolinska Directed Emotional Faces set (Lundqvist, Flykt, \& Ohman, 1998). Stimuli with unusual features (e.g., beards, glasses) were not used. Three sets (Sets 1, 2, and 3) of eight pictures (four males and four females) were used. Series of 16 faces were built by combining two sets (Series 12, 13, and 23). The order of presentation in each series was randomized. Then two versions (Versions 
A and B) were made of each series. In version A, eight faces expressed anger (four males and four females) and eight faces expressed happiness (four males and four females). In version $\mathrm{B}$, the facial expressions were reversed. A different combination (combinations 12A, 12B, 13A, 13B, 23A, and $23 B$ ) was used for each of the six classes selected in the school (e.g., combination 12A is version A of series 12). During the recognition phase, the three sets of faces (sets 1,2 , and 3) were combined to build a series of 24 neutral faces.

The task was programmed with E-prime to ensure accurate presentation times. During the encoding phase, faces were displayed one by one with a stimulus onset asynchrony of 5,500 ms. Each face was displayed for 5,000 ms followed by a blank screen for $500 \mathrm{~ms}$. In the recognition phase, faces were displayed with a question and two answer buttons at the bottom of the screen. The first question was "Is this a person you have seen before?" and the answer buttons were "Yes" and "No." If the participant answered "Yes" with the mouse, the question was replaced by "Did this face have a joyful expression or an angry expression when you saw it before?"

A countdown started at the end of the encoding phase and the recognition phase started automatically after $5 \mathrm{~min}$. Meanwhile, the adolescents filled in a general information questionnaire (gender, date of birth, etc.). The instructions indicated that faces would be displayed and the participants would have to recognize them again 5 min later. The instructions did not mention that the faces would display any emotion. Thus, the encoding of emotional information is considered to be implicit.

\section{Statistical analysis}

The acceptance or rejection of a hypothesis based on pvalue alone has been shown to be problematic, mainly because p-values do not distinguish effect size from sample size (Schmidt, 1996). We followed the recommendation of the Task Force on Statistical inference (Wilkinson, 1999) and calculated effect sizes within 95\% Confidence Intervals (CI). To estimate relationships between variables, we used Pearson's correlations (r). To estimate differences between groups, we used effect size correlations $\left(\mathrm{r}_{\text {effect size }}\right)$, an estimator developed by Rosenthal, Rosnow, and Rubin (2000). The advantage of this correlation approach is that all effect sizes are given on the same scale (from -1 to 1 ). According to Cohen (1988), correlation of between .10 and .30 (absolute value) corresponds to a small effect, between .30 and .50 to a moderate effect, and above .50 to a large effect. A correlation $<.10$ (absolute value) is considered to be negligible. Estimates are marked with an asterisk $\left(^{*}\right)$ if the 0 was not included in their $95 \% \mathrm{CI}$.
Assessing performance on a memory test by means of correctly detected old items (hits) is problematic because a participant who answers "old" to all old and new items will get the best score. Consequently, it is necessary to rely on signal detection theory and to calculate discrimination (d') and response bias (C). Discrimination is a better measure of memory performance because it is not confounded with the propensity to respond to all stimuli, that is, the response bias. Following the advice of Lockhart and Murdock (1970), we selected $\mathrm{C}$ rather than beta to measure the response bias. To do a signal detection analysis, both a target (e.g., old faces) and a response to the target (e.g., pressing the space bar) need to be defined. A hit is defined as a response to a target and a false alarm as a response to a nontarget (distractor). d' is a function of the difference between the number of hits and false alarms. For a subject who responds to all targets and distractors, this difference is null and that person's performance is poor. More generally, $\mathrm{d}^{\prime}=0$ indicates an inability to discriminate targets from distractors. $\mathrm{C}=0$ indicates the absence of response bias.

For the recognition of faces, a signal detection analysis was performed on "old" and "new" responses (Snodgrass \& Corwin, 1988). Old faces were defined as the targets. A high $d$ ' indicates a better ability to recognize old faces ( $d$ 'Face). A high $\mathrm{C}$ corresponds to a "conservative" attitude, that is, the participant is reluctant to answer "old" $\left(\mathrm{C}_{\text {Face }}\right)$. Then a similar signal detection analysis was performed, but only on responses to old angry versus new faces. A high d' indicates a better ability to recognize angry faces (d'Angry Face). Another analysis was performed on responses to old happy versus new faces. A high d' corresponds to a better ability to recognize happy faces ( $\mathrm{d}^{\text {'Happy Face }}$ ). A face recognition bias ( $\mathrm{d}_{\text {'Face Bias }}$ ) was assessed by the function d'Angry Face $-d$ ' Happy Face. Thus, a positive value indicates a better recognition of angry faces than happy faces. For the recall of facial expression, the signal detection analysis was performed on "angry" and "happy" responses, but only when the participant correctly recognized an old face. Angry expressions were defined as the target. A high d' indicates a better ability to discriminate

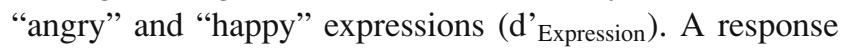
bias $\left(\mathrm{C}_{\text {Expression }}\right)$ was also calculated. $\mathrm{A}$ high $\mathrm{C}$ indicates a "conservative" attitude, that is, the participant is reluctant to answer "angry." One can define a memory bias as the tendency to memorize one given category of information better than another one. Based on this definition, it is important to note that the recognition bias (d'Face Bias) corresponds to a memory bias but that the response bias for expression doesn't $\left(\mathrm{C}_{\text {Expression }}\right)$. Details on the formulas and proofs used for the signal detection theory are available from the first author. All analyses were computed with R (R Development Core Team, 2006). 
Table 1 Comparison of Swiss $(n=86)$ and British $(n=3407)$ samples on the SDQ

\begin{tabular}{lllc}
\hline SDQ subscales & Swiss Mean $(S D)$ & British Mean $(S D)$ & $\mathrm{r}_{\text {effect size }}(95 \% \mathrm{CI})$ \\
\hline 1. Conduct problems & $1.36(1.83)$ & $0.9(1.7)$ & $.04^{*}(.01 .07)$ \\
2. Hyper./Inatt. & $3.29(2.89)$ & $2.6(2.7)$ & $.04^{*}(.01 .07)$ \\
3. Peer problems & $1.86(2.12)$ & $1.4(1.8)$ & $.04^{*}(.01 .07)$ \\
4. Emotional symptoms & $1.78(2.31)$ & $1.3(1.9)$ & $.04^{*}(.01 .07)$ \\
5. Prosocial behavior & $6.32(2.97)$ & $7.1(2.4)$ & $-.05^{*}(-.02-.08)$ \\
\hline
\end{tabular}

Note. Hyper./Inatt. = Hyperactivity/Inattention. Data from the British sample are taken from Meltzer, Gatward, Goodman, \& Ford (2000).

$* 0$ not included in the $95 \%$ confidence interval.

\section{Results}

Strengths and difficulties questionnaire

Means and SDs of the SDQ are presented in Table 1. These results were compared to the teacher SDQ norms reported for a British national survey of 3,407 adolescents aged from 11 to 15 years old (Meltzer, Gatward, Goodman, \& Ford, 2000). The mean difference between the Swiss and British samples for Conduct problems was significant but corresponded to a negligible effect, $r_{\text {effect size }}=.04^{*}$ (see equations 2.3 and 2.20 in Rosenthal, Rosnow, \& Rubin, 2000). The mean difference for Hyperactivity/Inattention was also negligible, $\mathrm{r}_{\text {effect size }}=.04^{*}$. This was also the case for Peer problems, $r_{\text {effect size }}=.04^{*}$, Emotional symptoms, $r_{\text {effect size }}=.04^{*}$, and Prosocial behavior, $\mathrm{r}_{\text {effect size }}=-.05^{*}$. Thus, even though means for problematic behavior are significantly higher in the Swiss sample than in the British one and the mean for prosocial behavior is lower, these differences are negligible in terms of effect size. In sum, we can consider that the observed behavior in the Swiss sample fell in the normal range.

Correlations between subscales showed that Conduct problems and Hyperactivity/Inattention were highly correlated, $r=.77^{*}$. The correlation between Emotional symptoms and Peer problems was large, $r=.55^{*}$. Conduct problems and Hyperactivity/Inattention were negatively related to Peer problems and Emotional symptoms, with small correlations (see Table 2). Prosocial behavior was mainly opposed to Conduct problems and Hyper-
activity/Inattention with large negative correlations. Conduct problems, Hyperactivity/Inattention, and Peer problems were more frequent among older adolescents, $r=.26^{*}$, $\mathrm{CI}=(.05, .45), r=.27^{*}, \mathrm{CI}=(.07, .46)$, and $r=.22^{*}$, $\mathrm{CI}=(.01, .41)$, respectively. These age effects were small in magnitude.

\section{Emotional face memory task}

The probability $(\mathrm{P})$ of responding "old" to an old face was $\mathrm{P}$ ("hit") $=.61$. The probability of responding "old" to a new face was $\mathrm{P}$ ("false-alarm") $=.18$. The probability of responding "angry" to an old angry face was $\mathrm{P}$ ("hit") $=.66$. The probability of responding "angry" to an old happy face was $\mathrm{P}$ ("false-alarm") $=.36$. A signal detection analysis was computed for the recognition of faces and the recall of expressions. Means and SDs are presented in Table 3. Adolescents were generally able to discriminate between old and new faces, as indicated by the positive d' value, $\mathrm{d}_{\text {Face }}=1.17^{*}, \mathrm{CI}=(1.04,1.30)$. They had a rather conservative attitude, that is, participants were generally reluctant to answer "old" when a face was presented, as indicated by the positive response bias index, $\mathrm{C}_{\text {Face }}=0.31^{*}, \mathrm{CI}=(0.23$, 0.38). Happy faces were better memorized than angry faces, as indicated by the negative value of the bias, d'Face Bias, $M=-0.24^{*}, \mathrm{CI}=(-0.39,-0.09)$. Participants were also able to discriminate "angry" from "happy" expressions, $\mathrm{d}^{\prime}$ Expression $=0.68^{*}, \mathrm{CI}=(0.51,0.84)$. The signal detection analysis also indicated that there was no response bias for expression, $\mathrm{C}_{\text {Expression, }} M=0.00, \mathrm{CI}=(-0.07,0.07)$.

Table 2 Correlation between the SDQ subscales (95\% CI)

\begin{tabular}{llllll}
\hline SDQ subscales & 1 & 2 & 3 & 4 & 5 \\
\hline 1. Conduct problems & - & - & - & - & - \\
2. Hyper./Inatt. & $.77^{*}(.67 .84)$ & - & - & - & - \\
3. Peer problems & $-.18(-.38 .03)$ & $-.18(-.38 .03)$ & - & - & - \\
4. Emotional symptoms & $-.23^{*}(-.42-.02)$ & $-.12(-.32 .10)$ & $.55^{*}(.39 .68)$ & - & - \\
5. Prosocial behavior & $-.56^{*}(-.69-.40)$ & $-.61^{*}(-.72-.45)$ & $-.14(-.34 .07)$ & $-.00(-.22 .21)$ & - \\
\hline
\end{tabular}

Note. Hyper./Inatt. = Hyperactivity/Inattention.

${ }^{*} 0$ not included in the $95 \%$ confidence interval. 
Table 3 Means $(S D)$ for the signal detection analysis

\begin{tabular}{lrl}
\hline Signal indices & Mean & $S D$ \\
\hline Face recognition & & \\
d' Face & $1.17^{*}$ & 0.62 \\
C $_{\text {Face }}$ & $0.31^{*}$ & 0.35 \\
d'Angry Face & $1.05^{*}$ & 0.78 \\
d'Happy Face & $1.29^{*}$ & 0.63 \\
d' ${ }_{\text {Face Bias }}$ & $-0.24^{*}$ & 0.68 \\
Expression recall & & \\
d'Expression & $0.68^{*}$ & 0.78 \\
C & 0.00 & 0.32 \\
\hline
\end{tabular}

Note. $\mathrm{d}_{\text {Face }}=$ recognition for faces, $\mathrm{C}_{\mathrm{Face}}=$ response bias for faces, $d^{\prime}$ Angry Face $=$ recognition for angry faces, $d^{\prime}$ Happy Face $=$ recognition for happy faces, $d^{\prime}$ Face Bias $=$ recognition bias for angry faces, $\mathrm{d}^{\text {Expression }}=$ discrimination between angry and happy expressions, $\mathrm{C}_{\text {Expression }}=$ response bias for expressions.

$* 0$ not included in the $95 \%$ confidence interval.

The ability to recognize old faces ( $\mathrm{d}^{\text {'Face }}$ ) decreased with age, $r=-.25^{*}, \mathrm{CI}=(-.44,-.04)$. Further analysis indicated that this developmental trend was due to an increase in false alarms with age. It was also found that older adolescents respond faster. Overall, these results suggest that older adolescents are more "impulsive" when they are asked to recognize faces. However, there was no significant correlation between age and the ability to discriminate expressions (d'Expression). Age also had no significant effects on the recognition bias ( $\mathrm{d}^{\text {'Face Bias }}$ ) or the response bias for expressions $\left(\mathrm{C}_{\text {Expression }}\right)$.

Correlations between behavior and memory for emotional faces

The results for the recognition of faces are presented in Table 4. Correlations indicated that Conduct problems were significantly related to a memory bias for angry faces, $r=.23^{*}$. This bias was mainly due to a better ability to recognize angry faces. Indeed, Conduct problems had a small positive correlation with the discrimination of angry faces (d'Angry Face), $r=.16$, and a negligible negative correlation with the discrimination of happy faces (d'Happy Face), $r=-.04$. Hyperactivity/Inattention was significantly related to a memory bias for angry faces, $r=.23^{*}$. As with Conduct problems, this bias was mainly due to a small positive correlation with the discrimination of angry faces (d'Angry Face), $r=.19$, and a negligible negative correlation with the discrimination of happy faces (d'Happy Face), $r=-.01$. The Peer problems factor was related to a memory bias against angry faces, $r=-.22^{*}$. The memory bias was due to a moderate negative correlation with the discrimination of angry faces, $r=-.34^{*}$, and a small negative correlation with the discrimination of happy faces, $r=-.18$. Peer problems were also significantly related to a reduced ability to recognize old faces (d'Face), $r=-.31^{*}$. Emotional symptoms were significantly related to a memory bias against angry faces, $r=-.35^{*}$. This bias was due to a small and negative correlation with the discrimination of angry faces, $r=-.28^{*}$, and a negligible positive correlation with the discrimination of happy faces, $r=.03$. Prosocial behavior was not significantly related to a recognition bias, $r=.01$. In sum, the effect size of the recognition bias was small for Conduct problems, Hyperactivity/Inattention and Peer problems, and moderate for Emotional symptoms.

The results for the recall of facial expressions are presented in Table 5. Correlations between adolescents' behavior and the ability to discriminate angry expressions (d'Expression $)$ or the response bias $\left(\mathrm{C}_{\text {Expression }}\right)$ were nonsignificant. It therefore seems that adolescents with problematic behavior do not have any difficulty remembering the expressions on faces they recognize correctly and that their responses are not biased.

\section{Moderating effect of impulsivity}

For all regressions, predictors were entered simultaneously because we were interested in assessing the relative importance of predictors rather than testing different models by selecting predictors (see Howell, 1998: 601-613). The memory bias regressed on the four facets of impulsivity, sex, and age revealed no significant result. This suggests that impulsivity has no main effect on the memory bias for angry faces. Regressions were then computed to test whether impulsivity

Table 4 Correlation between the recognition of emotional faces and behavior (95\% CI)

\begin{tabular}{|c|c|c|c|c|c|}
\hline SDQ subscales & $d_{\text {Face Bias }}$ & $\mathrm{d}_{\text {'Angry Face }}$ & $d_{\text {Happy Face }}$ & $d_{\text {Face }}$ & $\mathrm{C}_{\text {Face }}$ \\
\hline Conduct problems & $.23^{*}(.01 .42)$ & $.16(-.05 .36)$ & $-.04(-.25 .17)$ & $.07(-.14 .28)$ & $-.10(-.31 .11)$ \\
\hline Hyper./Inatt. & $.23^{*}(.02 .42)$ & $.19(-.02 .39)$ & $-.01(-.22 .20)$ & $.11(-.10 .32)$ & $-.11(-.32 .10)$ \\
\hline Peer problems & $-.22^{*}(-.41-.01)$ & $-.34^{*}(-.52-.14)$ & $-.18(-.38 \quad .03)$ & $-.31^{*}(-.49-.11)$ & $-.19(-.38 .03)$ \\
\hline Emotional symptoms & $-.35^{*}(-.52-.15)$ & $-.28 *(-.47-.07)$ & $.03(-.18 \quad .24)$ & $-.17(-.37 .05)$ & $-.04(-.25 .18)$ \\
\hline Prosocial behavior & $.01(-.20 \quad .23)$ & $.07(-.14 \quad .28)$ & $.08(-.14 .28)$ & $.09(-.12 .30)$ & $.17(-.05 \quad .37)$ \\
\hline
\end{tabular}

Note. $\mathrm{d}_{\text {'Face Bias }}=$ recognition bias for angry faces, d' Angry Face = recognition for angry faces, d' ${ }_{\text {Happy Face }}=$ recognition for happy faces, $\mathrm{d}^{\prime}$ Face $=$ recognition for faces, $\mathrm{C}_{\text {Face }}=$ response bias for faces.

${ }^{*} 0$ not included in the $95 \%$ confidence interval. 
Table 5 Correlation between the recall of emotional expressions and behavior $(95 \% \mathrm{CI})$

\begin{tabular}{lcc}
\hline SDQ subscales & \multicolumn{1}{l}{$\mathrm{d}^{\prime}$ Expression } & \multicolumn{1}{l}{$\mathrm{C}_{\text {Expression }}$} \\
\hline Conduct problems & $.14(-.07 .34)$ & $.14(-.08 .34)$ \\
Hyper./Inatt. & $.01(-.20 .22)$ & $.15(-.06 .35)$ \\
Peer problems & $.12(-.09 .33)$ & $.07(-.14 .28)$ \\
Emotional symptoms & $.14(-.07 .34)$ & $-.01(-.23 .20)$ \\
Prosocial behavior & $-.07(-.28 .14)$ & $-.15(-.35 .06)$ \\
\hline
\end{tabular}

Note. d'Expression $=$ discrimination between angry and happy expressions, $\mathrm{C}_{\text {Expression }}=$ response bias for expressions.

${ }^{*} 0$ not included in the $95 \%$ confidence interval.

did moderate the link between the recognition bias for angry faces and problematic behavior. Similar results were found when Conduct problems or Hyperactivity/Inattention was used as the criterion. To reduce the number of tables, results are presented for the sum of Conduct problems and Hyperactivity/Inattention. This sum can be considered as reflecting disruptive behavior. Disruptive behavior was regressed on the recognition bias, the four dimensions of impulsivity and their interactions with the bias. Gender and age were also entered as predictors. Gender was set to 0 for girls, and 1 for boys. Other predictors and the criteria were standardized in order to allow the interpretation of interaction effects (Aiken \& West, 1991). The results of a least square regression revealed 3 extreme residuals $(>2 * S D)$. To reduce their influence on the estimation, the regression was computed again with a robust estimator (M estimator, Venables, \& Ripley, 2002: 158). The results revealed a significant effect of the bias, beta $=0.29^{*}, \mathrm{CI}=(0.08,0.50)$, the bias $\times$ lack of Perseverance interaction, beta $=0.48^{*}, \mathrm{CI}=(0.15,0.80)$, and age, beta $=0.19^{*}, \mathrm{CI}=(0.01,0.38)$ (Table 6). These results indicated firstly that the effect of the recognition bias on disruptive behavior remained when impulsivity, gender, and age were taken into account. Secondly, the effect of the recognition bias on disruptive behavior was more pronounced for adolescents with a lack of Perseverance. When Peer problems or Emotional symptoms were used as criteria, there was no significant interaction between the bias for angry faces and the four facets of impulsivity, but the significant effect of the recognition bias remained.

In order to decompose the interaction effect, a model with only the memory bias as predictor and the lack of Perseverance as moderator was computed in order to predict disruptive behavior (Aiken \& West, 1991). The model was estimated for a low ( $-1 S D)$, average (0), and high $(+1 S D)$ level of lack of Perseverance. In this model, the effect of the lack of Perseverance was beta $=0.15, \mathrm{CI}=(-0.02,0.33)$ and the interaction effect was beta $=0.34^{*}, \mathrm{CI}=(0.18$, $0.50)$. For the low level of lack of Perseverance, the memory bias effect was beta $=-0.02, \mathrm{CI}=(-0.24,0.21)$. For the average level, the effect was $0.32^{*}, \mathrm{CI}=(0.14,0.50)$.
Table 6 Disruptive behavior regressed on memory bias, impulsivity, gender and age

\begin{tabular}{lclrl}
\hline Variable & \multicolumn{1}{l}{ Beta } & \multicolumn{1}{l}{$S E$} & \multicolumn{1}{l}{$t$} & $p$ \\
\hline (Intercept) & -0.29 & 0.16 & - & - \\
Bias & $0.29^{*}$ & 0.10 & 2.78 & .01 \\
Urg. & 0.16 & 0.10 & 1.61 & .11 \\
L. Prem. & 0.15 & 0.12 & 1.21 & .23 \\
L. Pers. & -0.00 & 0.13 & -0.04 & .97 \\
Sens. Seek. & -0.05 & 0.12 & -0.39 & .70 \\
Bias $\times$ Urg. & 0.07 & 0.12 & 0.59 & .56 \\
Bias $\times$ L. Prem. & -0.21 & 0.12 & -1.67 & .10 \\
Bias $\times$ L. Pers. & $0.48^{*}$ & 0.16 & 2.92 & .00 \\
Bias $\times$ Sens. Seek. & 0.03 & 0.09 & 0.35 & .73 \\
Sex & 0.21 & 0.21 & 1.01 & .31 \\
Age & $0.19^{*}$ & 0.09 & 2.08 & .04 \\
\hline
\end{tabular}

Note. Disruptive behavior $=$ Conduct problem + Hyperactivity/ Inattention. Urg. $=$ Urgency, L. Prem. $=$ Lack of Premeditation, L. Pers. $=$ Lack of Perseverance, Sens. Seek. $=$ Sensation seeking.

${ }^{*} 0$ not included in the $95 \%$ confidence interval.

For the high level (impulsive adolescents), the memory bias effect was beta $=0.66^{*}, \mathrm{CI}=(0.41,0.91)$. It can be concluded that for adolescents who have a good ability to control their impulses, the memory bias was not related to disruptive behavior (see Fig. 1).

\section{Discussion}

The first objective of this study was to see whether antisocial behavior in adolescents was associated with a memory bias for hostile information. Supporting the main hypothesis, the results showed that Conduct problems, which cover antisocial behavior, were related to a memory bias for angry faces. This bias was mainly due to a better recognition of angry faces. Previous studies have shown that children with trait anger or antisocial behavior paid more attention to anger-related information (e.g., Gouze, 1987). We can therefore make the hypothesis that adolescents with conduct problems allocate more attention to angry faces during the encoding phase of the memory task and that this attentional bias leads to a memory bias. However, the memory bias could also be due to a better consolidation and/or retrieval of anger-related information. For instance, in real-world situations, rumination may be a process by which anger-related episodes are consolidated in long-term memory (Rusting \& Nolen-Hoeksema, 1998). More generally, the memory bias for angry faces might be explained by an acquired hostile schema. Several empirical and theoretical considerations indicate that exposure to community or family violence favors the formation of a hostile schema that guides social information processing, favors attribution bias, and promotes aggressive responses (Dodge, Bates, \& Pettit, 1990). Such a hostile schema could also yield a memory bias for angry faces. An 
Fig. 1 Moderation effect of Perseverance on the relationship between the memory bias for angry faces and disruptive behavior (regression with standardized variables) important avenue for future research is to test whether a memory bias for angry faces is correlated to the attribution of hostile intentions and angry emotions. It would also be interesting to see whether a memory bias for angry faces is partly explained by past social experiences and knowledge stored in memory.

Like Conduct problems, Hyperactivity/Inattention was related to a memory bias for angry faces, mainly due to the better recognition of such faces. This result is not surprising because Conduct problems and Hyperactivity/Inattention are closely related (d'Acremont \& Van der Linden, 2006). Conduct disorder (CD) and Attention Deficit/Hyperactive Disorder (ADHD) are frequently comorbid and there has been some debate in the literature as to whether they are really separate conditions. In addition, CD and ADHD share common risk factors such as harsh parenting, and the development of a hostile schema may characterize both disorders (Patterson, DeGarmo, \& Knutson, 2000). Hyperactive/inattentive adolescents also experience more anger in daily life (Whalen, Jamner, Henker, Delfino, \& Lozano, 2002). A hostile schema or a mood-congruent effect could explain why adolescents with Hyperactive/Inattention problems showed a memory bias for angry faces in our study.

Emotional symptoms were related to a memory bias against angry faces. This bias was mainly due to a reduced ability to recognize angry faces. Thus Emotional symptoms and Conduct problems had opposing relationships with the recognition of angry faces. Research conducted in the field of cognitive neuroscience might help us to interpret this opposition. A large body of research has related anger to an asymmetric functioning of the right and left frontal regions
Moderating effect

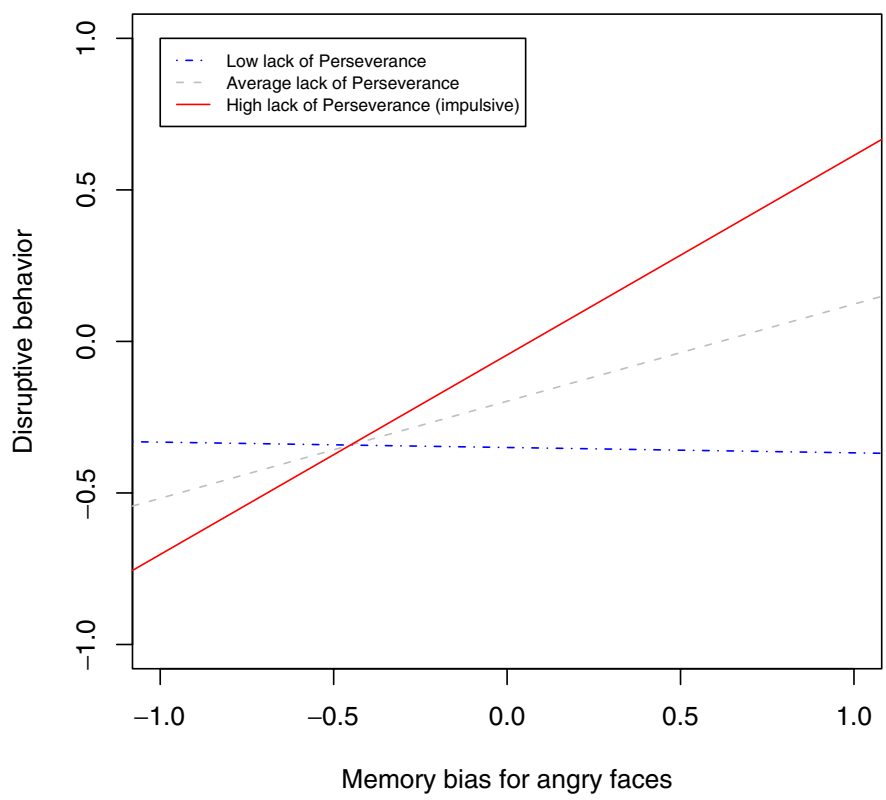

of the brain (Davidson, 2004). More specifically, it appears that an asymmetry in favor of the left frontal cortex supports approach-related emotions like anger while an asymmetry in favor of the right frontal cortex supports withdrawalrelated emotions like anxiety and sadness (Harmon-Jones \& Allen, 1998; Tutus, Kibar, Sofuoglu, Basturk, \& Gönül, 1998). Based on this evidence, one can propose the hypothesis that the observed link between Conduct problems and a memory bias in favor of angry faces reflects approach tendencies and is underlain by greater activity in the left frontal cortex. Conversely, the link between Emotional symptoms and a memory bias against angry faces may reflect avoidance tendencies and be underlain by greater activity in the right frontal cortex. These suggestions are speculative and should be explored empirically.

Concerning the other types of problematic behavior, Peer problems were related to a reduced ability to recognize emotional faces. This memory impairment was due to a poorer ability to recognize both happy and angry faces. Because the impairment was more pronounced for angry faces, Peer problems were also related to a bias against angry faces. An impaired memory for emotional faces, especially angry faces, may contribute to social maladjustment. Indeed, if an individual finds it difficult to recognize angry people, he/she might be more exposed to negative interactions with peers because of their unexpected negative reactions. Contrary to the four types of problematic behavior, Prosocial behavior was not related to a memory bias for angry or happy faces. On the whole, the memory bias for angry faces was found to be specifically related to Conduct problems and Hyperactivity/Inattention in a community sample of adolescents. 
The second aim of this study was to explore the relationships between impulsivity, memory for emotional faces, and problematic behavior in adolescence. We hypothesized that impulsivity would moderate the effect of a memory bias for angry faces. Regression analyses firstly showed that the memory bias for angry faces predicted Conduct problems and Hyperactivity/Inattention after impulsivity, gender, and age were controlled for. Secondly, the regressions indicated that one dimension of impulsivity, lack of Perseverance, moderated the memory bias effect. That is, the memory bias effect on Conduct problems and Hyperactivity/Inattention was more pronounced for adolescents with a lack of Perseverance. Perseverance may reflect resistance to proactive interference (Bechara \& Van der Linden, 2005), that is, the resistance to memory intrusions from information that was previously relevant to the task but has since become irrelevant. Resistance to proactive interference may also be impaired in persons with hyperactivity and inattention. Supporting this hypothesis, Cornoldi et al. (2001) have shown that children with hyperactivity and inattention symptoms experienced a higher number of intrusions during working memory tasks and concluded that these children were not capable of suppressing information that initially needed to be processed. Resistance to proactive interference may help adolescents to limit the interference of anger-related cognition during social interaction and thus reduce the effect of a memory bias.

Perseverance may also depend on sustained attention processes, which are involved in the capacity to maintain a sufficient attentional level in long-duration tasks. It is worth noting that studies of children have found that an impairment of sustained attention increases the effect of negative emotionality on social adjustment (Belsky, Friedman, \& Hsieh, 2001). It has been proposed that children with good attentional control are able to regulate negative emotions by turning their attention away from stimuli that generate frustration and anger (Eisenberg et al., 2000). Similarly, sustained attention could help adolescents to keep on with an ongoing activity or social interaction and to ignore people or situations that activate anger-related episodes in memory. In sum, sustained attention and resistance to proactive interference may explain why Perseverance moderates the effect of a memory bias for angry faces during social interaction. In line with this latter interpretation, we propose that only impulsive adolescents with a memory bias will act upon their biased representations whereas less impulsive adolescents will not.

This study has several limitations. First of all, the sample size was rather small and replication studies with larger sample sizes are necessary to increase the precision of the estimates. To increase the psychometric quality of the behavior assessment, it would be appropriate to use a multi-informant method. It should also be noted that the psychometric quali- ties of the emotional face memory task are as yet unknown. The reliability and validity of this procedure should be verified in future studies. The design of our study is correlational. Consequently, it impossible to decide whether a memory bias for angry faces causes conduct problems (among other causes), whether conduct problems cause a memory bias, or whether their relationship is explained by a third variable, for instance knowledge stored in memory. In order to find causal links, longitudinal and experimental designs are needed.

In conclusion, this study showed that conduct problems and Hyperactivity/Inattention in adolescence are related to a memory bias for angry faces, mainly due to a better recognition of angry faces. These results are new and suggest that memory processes for emotional stimuli play an important role in disruptive behavior during adolescence. The effect of the memory bias for angry faces was moderated by one specific aspect of impulsivity: lack of Perseverance. This result suggests that attentional control can help adolescents to reduce the influence of anger-related memories.

Acknowledgements This research was supported by the Swiss National Centre of Competence in Research (NCCR) "Affective sciences: Emotions in individual behaviour and social processes." We thank the administration of the Geneva public schools who gave their authorization for the research. We also thank Michelle Bishoff, Laura Bless, Mena Dell'Armin, and Cécile Grangier for their help in collecting the data.

\section{References}

d'Acremont, M. (2005). Les conduites antisociales chez l'adolescent: Le rôle de l'impulsivité, des capacités de prise de décision, et des biais cognitifs [Antisocial behavior in adolescence: The role of impulsivity, decision-making, and cognitive biases]. Unpublished doctoral dissertation, University of Geneva, Geneva.

d'Acremont, M., \& Van Der Linden, M. (2005). Adolescent impulsivity: Findings from a community sample. Journal of Youth and Adolescence, 34, 427-435.

d'Acremont, M., \& Van Der Linden, M. (2006). Confirmatory factor analysis of the Strengths and Difficulties Questionnaire in a Community Sample of French-Speaking Adolescents. Manuscript under revision.

Aiken, L. S., \& West, S. G. (1991). Multiple regression: Testing and interpreting interactions. California: Sage Publications.

Bechara, A., \& Van Der Linden, M. (2005). Decision-making and impulse control after frontal lobe injuries. Current Opinion in Neurology, 18, 734-739.

Belsky, J., Friedman, S. L., \& Hsieh, K. H. (2001). Testing a core emotion-regulation prediction: Does early attentional persistence moderate the effect of infant negative emotionality on later development. Child Development, 72, 123-133.

Bruce, V., \& Young, A. (1986). Understanding face recognition. British Journal of Psychology, 77, 305-327.

Cohen, J. (1988). Statistical power analysis for the behavioral sciences. Hillsdale, NJ: Erlbaum.

Colder, C. R., \& Stice, E. (1998). A longitudinal study of the interactive effects of impulsivity and anger on adolescent problem behavior. Journal of Youth and Adolescence, 27, 255-274. 
Cornoldi, C., Marzocchi, G. M., Belotti, M., Caroli, M. G., De Meo, T., \& Braga, C. (2001). Working memory interference control deficit in children referred by teachers for ADHD symptoms. Child Neuropsychology, 7, 230-240.

Crick, N. R., \& Dodge, K. A. (1994). A review and reformulation of social information processing mechanisms in children's social adjustment. Psychological Bulletin, 115, 74-101.

D'Argembeau, A., Van Der Linden, M., Etienne, A. M., \& Comblain, C. (2003). Identity and expression memory for happy and angry faces in social anxiety. Acta Psychologica, 114, 1-15.

Davidson, R. J. (2004). What does the prefrontal cortex "do" in affect: Perspectives on frontal EEG asymmetry research. Biological Psychology, 67, 219-233.

Dodge, K. A., Bates, J. E., \& Pettit, G. S. (1990). Mechanisms in the cycle of violence. Science, 250, 1678-1683.

Dodge, K. A., \& Frame, C. L. (1982). Social cognitive biases and deficits in aggressive boys. Child Development, 53, 620-635.

Dodge, K. A., Laird, R., Lochman, J. E., Zeli, A., \& Conduct Problems Prevention Research Group. (2002). Multidimensional latentconstruct analysis of children's social information processing patterns: Correlations with aggressive behavior problems: The clinical assessment of children and adolescents. Psychological Assessment, 14, 60-73.

Dodge, K. A., \& Newman, J. P. (1981). Biased decision-making processes in aggressive boys. Journal of Abnormal Psychology, 90, $375-379$.

Eisenberg, N., Guthrie, I. K., Fabes, R. A., Shepard, S., Losoya, S., Murphy, B. C., et al. (2000). Prediction of elementary school children's externalizing problem behaviors from attention and behavioral regulation and negative emotionality. Child Development, $71,1367-1382$.

Fombonne, E., d'Acremont, M., Van Der Linden, M., Capron, C., Maufroy, M., \& Proust, L. (2005). Questionnaire Points fortsPoints faibles (SDQ-Fra) [Strengths and Difficulties Questionnaire: One-sided informant-rated version for teachers and parents of 4-16 year olds]. Retrieved from http://www.sdqinfo. $\mathrm{com} / \mathrm{d} 8 . \mathrm{html}$

Gerbing, D. W., Ahadi, S. A., \& Patton, J. H. (1987). Toward a conceptualization of impulsivity: Components across the behavioral and self-report domains. Multivariate Behavioral Research, 22, $357-379$.

Goodman, R. (2001). Psychometric properties of the strengths and difficulties questionnaire. Journal of the American Academy of Child and Adolescent Psychiatry, 40, 1337-1345.

Goodman, R. (1997). The strengths and difficulties questionnaire: A research note. Journal of Child Psychology and Psychiatry, 38, $581-586$

Gouze, K. R. (1987). Attention and social problem solving as correlates of aggression in preschool males. Journal of Abnormal Child Psychology, 15, 181-197.

Harmon-Jones, E., \& Allen, J. J. B. (1998). Anger and frontal brain activity: EEG asymmetry consistent with approach motivation despite negative affective valence. Journal of Personality and Social Psychology, 74, 1310-1316.

Howell, D. (1998). Méthodes statistiques en sciences humaines [Statistical methods for psychology]. Paris: De Boeck Université.

Lochman, J. E., \& Dodge, K. A. (1994). Social-cognitive processes of severely violent, moderately aggressive, and nonaggressive boys. Journal of Consulting Clinical Psychology, 62, 366-374.

Lockhart, R. S., \& Murdock, B. B. (1970). Memory and the theory of signal detection. Psychological Bulletin, 74, 100-109.

Luengo, M. A., Carrillo-de la-Pena, M. T., Otero, J. M., \& Romero, E. (1994). A short-term longitudinal study of impulsivity and antisocial behavior. Journal of Personality and Social Psychology, 66, 542-548.

Lundqvist, D., Flykt, A., \& Öhman, A. (1998). Karolinska directed emotional faces set [Data file]. Stockholm: Department of Neurosciences, Karolinska Hospital.

MacCallum, R. C., Zhang, S., Preacher, K. J., \& Rucker, D. D. (2002). On the practice of dichotomization of quantitative variables. $P s y$ chological Methods, 7, 19-40.

Meltzer, H., Gatward, R., Goodman, R., \& Ford, T. (2000). Mental health of children and adolescents in Great Britain. Retrieved from http://www.sdqinfo.com/b8.html. London: The Stationery Office.

Milich, R., \& Dodge, K. A. (1984). Social information processing in child psychiatric populations. Journal of Abnormal Child Psychology, 12, 471-489.

Orobio-de Castro, B., Veerman, J. W., Koops, W., Bosch, J. D., \& Monshouwer, H. J. (2002). Hostile attribution of intent and aggressive behavior: A meta-analysis. Child Development, 73, 916934.

Patterson, G. R., DeGarmo, D. S., \& Knutson, N. (2000). Hyperactive and antisocial behaviors: Comorbid or two points in the same process. Development and Psychopathology, 12, 91-106.

R Development Core Team (2006). R: A language and environment for statistical computing (Version 2.3.0) [Computer software]. Retrieved from http://www.r-project.org. Vienna, Austria: R Foundation for Statistical Computing.

Rosenthal, R., Rosnow, R., \& Rubin, D. (2000). Contrasts and effect sizes in behavioral research: A correlation approach. Cambridge: Cambridge University Press.

Rusting, C. L., \& Nolen-Hoeksema, S. (1998). Regulating responses to anger: Effects of rumination and distraction on angry mood. Journal of Personality and Social Psychology, 74, 790803.

Schafer, J. L., \& Graham, J. W. (2002). Missing data: Our view of the state of the art. Psychological Methods, 7, 147-177.

Schermelleh-Engel, K., \& Moosbrugger, H. (2003). Evaluating the fit of structural equation models: Test of significance and descriptive goodness-of-fit measures. Methods of Psychological Research Online, 8, 23-74.

Schippell, P. L., Vasey, M. W., Cravens-Brown, L. M., \& Bretveld, R. A. (2003). Suppressed attention to rejection, ridicule, and failure cues: A unique correlate of reactive but not proactive aggression in youth. Journal of Clinical Child and Adolescent Psychology, $32,40-55$.

Schmidt, F. L. (1996). Statistical significance testing and cumulative knowledge in psychology: Implications for training of researchers. Psychological Methods, 1, 115-129.

Schultz, D., Izard, C. E., \& Bear, G. (2004). Children's emotion processing: Relations to emotionality and aggression. Development and Psychopathology, 16, 371-387.

Slaby, R. G., \& Guerra, N. G. (1988). Cognitive mediators of aggression in adolescent offenders: 1. Assessment. Developmental Psychology, 24, 580-88.

Snodgrass, J. G., \& Corwin, J. (1988). Pragmatics of measuring recognition memory: Applications to dementia and amnesia. Journal of Experimental Psychology: General, 117, 34-50.

Teichner, G., \& Golden, C. J. (2000). The relationship of neuropsychological impairment to conduct disorder in adolescence: A conceptual review. Aggression and Violent Behavior, 5, 509-528.

Tutus, A., Kibar, M., Sofuoglu, S., Basturk, M., \& Gönül, A. S. (1998). A technetium-99m hexamethylpropylene amine oxime brain single-photon emission tomography study in adolescent patients with major depressive disorder. European Journal of $\mathrm{Nu}$ clear Medicine and Molecular Imaging, 25, 601-606.

Van der Linden, M., d'Acremont, M., Zermatten, A., Jermann, F., Laroi, F., Willems, S., et al. (2006). A French adaptation of the UPPS impulsive behavior scale: Confirmatory factor analysis in a sample of undergraduate students. European Journal of Psychological Assessment, 22, 38-42. 
Venables, W. N., \& Ripley, B. D. (2002). Modern applied statistics with $S$. New-York: Springer.

Webster-Stratton, C., \& Lindsay, D. W. (1999). Social competence and early-onset conduct problems: Issues in assessment. Journal of Child Clinical Psychology, 28, 25-93.

Whalen, C. K., Jamner, L. D., Henker, B., Delfino, R. J., \& Lozano, J. M. (2002). The ADHD spectrum and everyday life: Experience sampling of adolescent moods, activities, smoking, and drinking. Child Development, 73, 209-227.
Whiteside, S. P., \& Lynam, D. R. (2001). The Five Factor Model and impulsivity: Using a structural model of personality to understand impulsivity. Personality and Individual Differences, 30, 669689.

Wilkinson, L. (1999). Statistical methods in psychology journals: Guidelines and explanations. American Psychologist, 54, 594604.

Zermatten, A., Van der Linden, M., d'Acremont, M., Jermann, F., \& Bechara, A. (2005). Impulsivity and decision making. The Journal of Nervous and Mental Disease, 193, 647-650. 